\title{
Masas hojaldradas fermentadas: croissant
}

\author{
Sourdough puff pastry: croissant \\ Alexia Soberanes Hernández ${ }^{a}$, Blanca Azalia López Hernández ${ }^{b}$, Mauricio Piñón Vargas ${ }^{c}$
}

\begin{abstract}
:
Sourdough puff pastry meets puff pastry and sourdough characteristics and both contribute in flavor and texture to the final product. The croissant -which means rising or growing-is a type of bread made out of this pastry. Its preparation may be easy but the process should be done the right way in order to obtain a great product.
\end{abstract}

\section{Keywords:}

Sourdough puff pastry, croissant, preparation

\begin{abstract}
Resumen:
Las masas hojaldradas fermentadas reúnen las características tanto de masas hojaldradas como de masas fermentadas, estas contribuyen con sabor y textura en el producto final. El croissant -del francés creciente- es una pieza de bollería hecha a base de este tipo de masa. La preparación de este platillo puede ser sencilla, pero es necesario que el proceso se haga de la manera correcta para obtener un buen producto.
\end{abstract}

\section{Palabras Clave:}

Masas hojaldradas fermentadas, croissant, preparación

\section{Introducción}

Las masas hojaldradas fermentadas son una combinación de la masa hojaldre y la masa con levadura. Para su preparación es necesario tener una masa básica leudante la cual se empasta con mantequilla o margarina y con la ayuda de un rodillo se estira cuidadosamente para evitar que la grasa se derrame. Una vez estirada se dobla en tres o cuatro partes -vuelta doble o triple- y se procede a estirar y doblar nuevamente para así formar los famosos pliegues que le otorgaran alveolado después de fermentar [1]. Este tipo de masa, aunque sencilla es bastante técnica, por lo que se necesita de mucha práctica para obtener un producto estético [2].

Una de las elaboraciones más conocidas realizada a partir de la masa anteriormente mencionada es el croissant -también conocido como cruasán, medialunas, cuernitos, etc- que, aunque se tenga la creencia de que es un producto nacido en Francia no lo es, su origen es austriaco [3]. Como es bien sabido, Austria es un país que destaca desde hace siglos por su repostería y panadería, en especial por platillos como la tarta Sacher, el apfelstrudel o la tarta Linzer y no es extraño que un platillo como el croissant tenga este origen [4]. El kipferl es una pieza de bollería en forma de medialuna hecha desde el siglo XIII, servida sola o algunas veces con nueces [5]. A pesar de que se conoce muy poco sobre su composición original hoy en día se preparan con ingredientes como leche, harina, azúcar, mantequilla y sal, pero a comparación del croissant francés, estos no se elaboran con masas hojaldradas.

Su introducción a Francia puede no ser muy acertada, ya que algunas fuentes aseguran que

\footnotetext{
a Alumna del programa educativo de la Licenciatura en Gastronomía de la Universidad Autónoma del Estado de Hidalgo, Instituto de Ciencias Económico - Administrativas, https://orcid.org/0000-0002-4848-5145,Email: so270269@uaeh.edu.mx

b Autor de Correspondencia, Profesora de Asignatura adscrita a la Licenciatura en Gastronomía de la Universidad Autónoma del Estado de Hidalgo,
Instituto de Ciencias Económico - Administrativas, https://orcid.org/0000-0003-4238-1246, Email: blanca_lopez@uaeh.edu.mx
c Profesor Investigador de Tiempo Completo adscrito a la Licenciatura en Gastronomía de la Universidad del Valle de Puebla, https://orcid.org/0000-
} 
María Antonieta fue quién los presentó ante la corte de Versalles mientras que otros dicen que fueron personas de origen austriaco las que introdujeron el kipferl en una panadería entre 1837 y 1839 . De la misma manera, la palabra croissant apareció por primera vez en diccionarios y libros en el año 1863.

Existe evidencia de que a principios del siglo XX los panaderos comenzaron a usar la masa hojaldrada para la preparación de los croissants y de manera innovadora agregaron levadura, creando así una masa aireada, suave, crocante y rica en sabor gracias a la mantequilla.

Durante el siglo XVIII el croissant era un aperitivo que solo cierta clase social podía consumir, después de la Primera Guerra Mundial este se hizo más accesible y asequible. Hoy en día se conocen dos tipos de croissant, el croissant au beurre -hecho con mantequilla- y el croissant ordinaire -hecho con margarina-, depende del tipo de grasa usada la forma que se le dará al producto.

Así como en países europeos, en países de América latina como Uruguay, Argentina y Paraguay existen variantes de este pan -medialunas- que incluso son consideradas típicas de algunas regiones y suelen prepararse con grasa animal como la manteca de vaca que le da un sabor distinto al croissant. En Venezuela y Ecuador se les llama cachitos. En Cuba y Perú este bocadillo se rellena con jamón, queso, chocolate y otros productos tradicionales de las zonas [6].

\section{Desarrollo}

a) Objetivo general de la práctica

Realizar la preparación de croissants a partir de una masa hojaldrada fermentada para reconocer su estructura y formado.

\section{b) Instrumentos necesarios}

Bowl, báscula gramera, rodillo, cuchillo o cortador de pizza, charola, brocha de silicón

c) Nombre de la práctica

\section{Croissants}

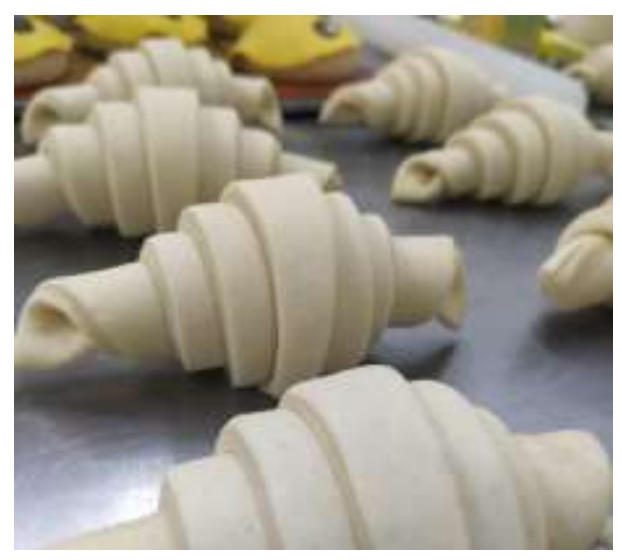

Ilustración 1. Formado de croissants [7]
Insumos requeridos para su realización

Tabla 1. Insumos para la preparación de croissants.

\begin{tabular}{|l|c|c|}
\hline \multicolumn{1}{|c|}{ Ingredientes } & Cantidad & Unidad \\
\hline Harina de trigo & 0.400 & $\mathrm{Kg}$ \\
\hline Sal & .0 .008 & $\mathrm{Kg}$ \\
\hline Azúcar & 0.050 & $\mathrm{Kg}$ \\
\hline Leche & 0.230 & $\mathrm{~L}$ \\
\hline $\begin{array}{l}\text { Levadura en } \\
\text { polvo }\end{array}$ & 0.009 & $\mathrm{Kg}$ \\
\hline $\begin{array}{l}\text { Mantequilla } \\
\text { Mantequilla(emp } \\
\text { aste) }\end{array}$ & 0.225 & $\mathrm{Kg}$ \\
\hline Huevo(barnizar) & 1 & $\mathrm{Pza}$ \\
\hline
\end{tabular}

d) Procedimiento Mise en place

1. Pesar los ingredientes correctamente.

2. Meter la mantequilla del empaste en una bolsa y con ayuda de un rodillo extender hasta formar un cuadro. Reservar.

\section{Procedimiento}

1. En un bowl mezclar harina, azúcar. sal, levadura, y leche hasta formar una masa. Agregar la mantequilla a temperatura ambiente y amasar hasta que tenga una consistencia suave. Con ayuda del rodillo extender la masa hasta formar un cuadro.

2. Sacar la mantequilla del empaste de la bolsa y poner sobre la masa extendida, cubrir y sellar.

3. Extender con mucho cuidado la masa evitando que la mantequilla se desbordehasta formar un rectángulo. Doblar en forma de folleto y extender nuevamente. Repetir el proceso dos veces más.

4. Después de empastar, extender la masa para cortar en triángulos con el cortador de pizza o cuchillo y proceder a formar los croissants. Para ello se toma uno de los triángulos de masa, se estira un poco y se enrolla en sí mismo para darle la forma conocida.

5. En una charola previamente engrasada se acomodan de tal manera que al crecer no se peguen unos con otros. Dejar reposar a temperatura ambiente un mínimo de dos horas o hasta que haya doblado su tamaño.

6. Barnizar con huevo y hornear a $200^{\circ} \mathrm{C}$ hasta que estén dorados. Sacar del horno y enfriar a temperatura ambiente. 


\section{Conclusión}

Se concluye que el croissant es una preparación que, aunque parezca simple requiere de una gran disciplina para obtener un buen producto y a pesar de que existen diferentes variedades y presentaciones del mismo, por medio de su preparación con una masa fermentada hojaldrada su textura y formado mantienen la esencia del producto y así mismo se adapta a las costumbres, tradiciones y sabores europeos.

\section{Referencias}

[1] Escuela Superior de Turismo \& Instituto Politécnico Nacional. (2012, 10 06). El origen, métodos y técnicas en masas laminadas y hojaldradas. IPN. Retrieved 08 13, 2021, from https://tesis.ipn.mx/bitstream/handle/123456789/23048/EL\%20ORIGE N\%2C\%20M\%C3\%89TODOS\%20Y\%20T\%C3\%89CNICAS\%20EN $\% 20$ MASAS $\% 20$ LAMINADAS $\% 20$ Y\% 20 HOJALDRADAS.pdf?sequ ence $=1 \&$ isAllowed $=\mathrm{y}$

[2] Diario de gastronomía. (2016, 03 07). Croissant, el rey del desayuno. Diario de Gastronomía. Retrieved 08 13, 2021, from https://diariodegastronomia.com/croissant-el-rey-del-desayuno/

[3] La Vanguardia. (2021, 01 30). Día Internacional del Croissant: la curiosa historia sobre su origen. La Vanguardia. Retrieved 08 13, 2021, from https://www.lavanguardia.com/comer/tendencias/20210130/6201492/di a-internacional-croissant-historico-origen-famoso-panecillo.html

[4] del Campo, M. (2011, 11 19). Austria y sus postres. El blog de infoidiomas. Retrieved 08 13, 2021, from https://www.infoidiomas.com/blog/2994/austria-y-sus-postres/

[5] Traub, C. (2021, 04 27). The Curious History of the Croissant (\& How It Became France's Favorite Pastry). Paris Unlocked. Retrieved 08 13, 2021, from https://www.parisunlocked.com/food/foodhistory/history-of-the-croissant-how-france-adopted-i

[6] Yen, J. (2021). Types of Croissants Across the Globe. Miss Buttercup. $\quad$ Retrieved 08 13, 2021, from https://www.missbuttercup.com/types-of-croissants/

[7] Autoría propia 